NASA/TM-2001-210762

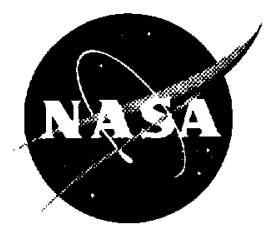

\title{
Development of a Hybrid RANS/LES Method for Compressible Mixing Layer Simulations
}

Nicholas J. Georgiadis

Glenn Research Center, Cleveland, Ohio

J. Iwan D. Alexander and Eli Reshotko

Case Western Reserve University, Cleveland, Ohio

Prepared for the

39th Aerospace Sciences Meeting and Exhibit

sponsored by the American Institute of Aeronautics and Astronautics

Reno, Nevada, January 8-11, 2001

National Aeronautics and

Space Administration

Glenn Research Center 
Available from

NASA Center for Aerospace Information 7121 Standard Drive

National Technical Information Service 5285 Port Royal Road Hanover, MD 21076 


\title{
Development of a Hybrid RANS/LES Method for Compressible Mixing Layer Simulations
}

\author{
Nicholas J. Georgiadis* \\ NASA Glenn Research Center, Cleveland, Ohio 44135 \\ J. Iwan D. Alexander ${ }^{\dagger}$ and Eli Reshotko ${ }^{\ddagger}$ \\ Case Western Reserve University, Cleveland, Ohio 44106
}

\begin{abstract}
A hybrid method has been developed for simulations of compressible turbulent mixing layers. Such mixing layers dominate the flows in exhaust systems of modern day aircraft and also those of hypersonic vehicles currently under development. The hybrid method uses a Reynolds-averaged Navier-Stokes (RANS) procedure to calculate wall bounded regions entering a mixing section, and a Large Eddy Simulation (LES) procedure to calculate the mixing dominated regions. A numerical technique was developed to enable the use of the hybrid RANS-LES method on stretched, non-Cartesian grids. The hybrid RANS-LES method is applied to a benchmark compressible mixing layer experiment. Preliminary two-dimensional calculations are used to investigate the effects of axial grid density and boundary conditions. Actual LES calculations, performed in three spatial directions, indicated an initial vortex shedding followed by rapid transition to turbulence, which is in agreement with experimental observations.
\end{abstract}

\section{Introduction}

The use of computational fluid dynamics (CFD) to assist in the analysis and design of aerospace vehicles and their components has substantially increased in recent years. For analyzing one particular class of flows, that of aircraft engine exhaust nozzles, Reynoldsaveraged Navier-Stokes (RANS) codes have been used extensively by government organizations (i.e. NASA) and aerospace companies. Exhaust nozzles being developed for modern day commercial aircraft typically have multiple streams with a core flow and one or more bypass streams which mix with the high energy core flow before exiting the nozzle to lower jet noise while maintaining high thrust levels. Similarly in NASA's recent High-Speed Research program, the engine exhaust systems for this proposed supersonic transport were designed to be mixer-ejector nozzles, which entrain secondary air into the exhaust nozzle to mix with the core engine stream, again with the goal of simultaneously lowering jet noise and maintaining sufficient thrust. ${ }^{1}$ Propulsion systems currently under development for use on hypersonic and reusable space launch vehicles, such as the Turbine-Based Combined-Cycle (TBCC) and Rocket-Based Combined-Cycle (RBCC) concepts also employ mixer ducts.

The flows in these nozzle systems all have compressible turbulent mixing as the dominant flow character-

\footnotetext{
* Aerospace Engineer, Nozzle Branch, Senior Member AlAA

${ }^{\dagger}$ Assoc. Professor, Department of Mechanical and Aerospace Engineering, Member AIAA

${ }^{\ddagger}$ Kent H. Smith Professor Emeritus, Department of Mechanical and Aerospace Engineering, Fellow AIAA
}

istic. RANS codes used by research and development engineers to analyze these nozzles have employed turbulence models to replace the unsteady turbulent motion with an effective eddy viscosity. Unfortunately, no turbulence model has been developed to date which is able to accurately represent the turbulent motion for such nozzle flows. Validation studies ${ }^{2,3}$ have shown that the "state of the art" turbulence models available in production-use RANS codes have major deficiencies in predicting turbulent mixing in nozzle and jet flows involving compressibility, high temperatures, and three-dimensionality.

The known limitations of RANS techniques to calculate complex turbulent flows, coupled with continually increasing computing power, have led to interest in more sophisticated calculation techniques such as direct numerical simulation (DNS) and large eddy simulation (LES). DNS is currently limited by computer hardware to very simple flows at low Reynolds numbers, and LES, which directly solves for the large turbulent scales and limits empirical modeling to the smallest scales, is becoming practical for more complex flows at higher Reynolds numbers. Birch ${ }^{4}$ and Bradshaw ${ }^{5}$ suggest that LES techniques offer the best prospects for improving the capability to calculate turbulent flows, particularly for flow regions not including wall boundary layers.

As a result, an LES-based technique is an attractive option for calculating the mixing dominated regions of nozzle flows. However, applying such an LES technique simultaneously to the wall bounded regions that enter the mixing region (which are an important part 
of multi-stream nozzles that should be calculated accurately) will not be practical in the near future. This is because computational resources far greater than those available today would be required to capture the wide range of turbulent time and length scales that are important in such a problem. These turbulent scales range from very small eddies in the wall boundary layers to very large eddies in the developing mixing layer.

Vearly all LES simulations of jet and mixing layer flows performed to date have placed the inflow of the computational domain downstream of any wall bounded regions and have either ignored the upstream boundary layer efferts or used some approximation to initialize the turbulent mixing layer. Several authors, such as Ragab ${ }^{6,7}$ and Hedges ${ }^{8}$ have imposed hyperbolic tangent mean velority profiles at the plane which represents the end of the wall boundary layer regions and the beginning of the mixing region. Ragab then used the results of a linear stability analysis to generate perturbations about the mean velocity profile located at the mixing plane. Hedges added small amplitude perturbations to the vertical velocity component in simulations of heated jet flows.

The difficulty in using an artificially generated inflow, such as that assuming a hyperbolic tangent velocity profile, is that the characteristics of the upstream boundary layers, including velocity, temperature, and turbulence profiles, are not accurately represented. This is a significant deficiency since the state of the incoming boundary layers have been shown to have substantial effects on the development of turbulent mixing layers in the experiments conducted by Bradshaw. Browand and Latigo, ${ }^{10}$ and Hussain and Clark. ${ }^{11}$

While RANS-based methods have major deficiencies in predicting compressible mixing layers and inherently are not formulated for calculation of unsteady turbulent flows, they have been shown to predict the mean flow behavior of wall bounded regions quite well, particularly in the absence of adverse pressure gradients. As a result, it would be desirable to combine a RANS-based technique for the wall boundary layers upstream of the mixing region with an LES-based technique for the downstream unsteady, turbulent mixing region. The development of such a hybrid RANS/LES approach is the subject of this work.

The method developed here is proposed as an alternative computational technique to performing LES calculations everywhere in the computational domain, that includes the mean flow characteristics of the incoming boundary layers and is also feasible when considering foreseeable computational resources.

\section{Hybrid RANS-LES Methods}

The realization that $\mathrm{LES}$ calculations of flows in aerospace and industrial applications at realistic Reynolds numbers will not be possible for some time has led to interest into the development of hybrid techniques. The objective of a hybrid method is to retain the essential features of the LES method, but to employ a computationally cheaper RANS method in regions where it is appropriate. As a result, nearly all hybrid methods proposed to date apply a RANS approach to attached wall boundary layer regions and an LES approach to regions of large scale separation. The work detailed in this paper represents the first hybrid method development for application to compressible mixing layers.

The most widely publicized hybrid method to date is the Detached Eddy Simulation (DES) method of Spalart. ${ }^{12-14}$ In the DES method, the wall bounded regions are calculated using RANS with the SpalartAllmaras ${ }^{15}$ one equation turbulence model. Constantinescu and Squires ${ }^{16}$ have applied Spalart's DES method to turbulent flow over a sphere. which is an appropriate geometry for the method due to the large scale separation in the wake of the sphere.

Speziale $^{17}$ suggested an approach that allows for computations varying from RANS in the coarse grid limit, through LES, and finally to DNS in the very fine grid limit. A Reynolds Stress model is used to close the turbulent stresses in the RANS limit, and provides the basis for a subgrid model necessary in LES simulations. Batten et al. ${ }^{18}$ also propose a hybrid model that employs a Reynolds-Stress model to close the RANS and LES equations. Lastly, Arunajatesan et al. ${ }^{19}$ have applied a hybrid RANS-LES method to cavity flowfields. Their approach employed a two-equation $k-k l$ turbulence model to close the RANS equations and a one-equation model solving for the filtered subgrid kinetic energy to close the LES equations.

The hybrid RANS-LES method presented here is developed for application to configurations such as the mixing layer shown in figure 1. This relatively simple configuration is representative of more complex nozzle geometries in that two wall bounded regions provide isolated flows to a single region where compressible mixing is the primary flow characteristic. Development of the hybrid method and preliminary assessment of the method for a benchmark compressible mixing layer configuration are the focus of this paper.

The hybrid method employs a RANS approach to provide the mean flow characteristics of the wall boundary layers entering the mixing region. The downstream mixing layer is then calculated using LES. The method developed here is intended for those nozzle and mixing layer problems in which a geometric feature, such as the base region of a nozzle or splitter plate separating the upstream flows, provides the dominant unsteady mechanism to drive the development of turbulence in the mixing layer. Although the upstream RANS approach does not provide any unsteady turbulent information to the mixing layer, the mean flow momentum and thermal boundary layer effects are calculated and provided to the LES region. 


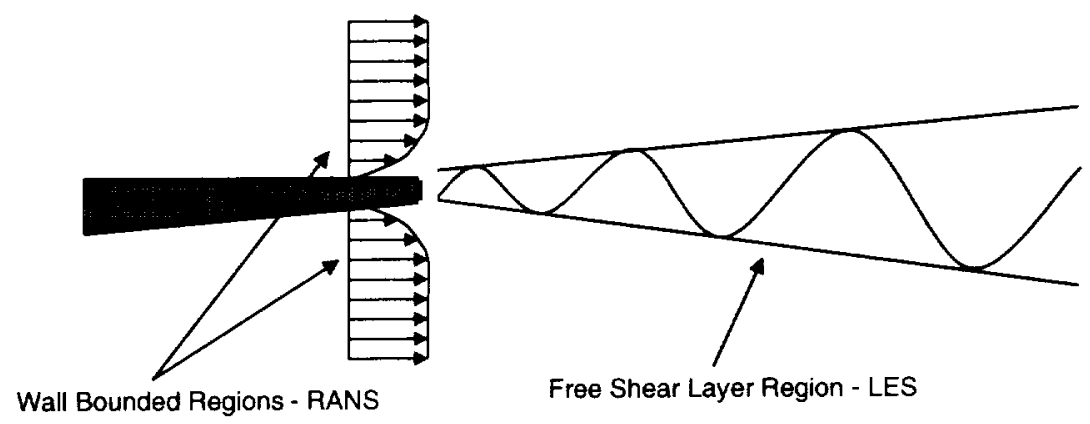

Fig. 1 Schematic of mixing layer demonstrating the hybrid RANS/LES approach

\section{Method Formulation}

Both the RANS and LES equations are derived starting from the general form of the Navier-Stokes equations, written in tensor form. The expression for conservation of mass, or the continuity equation is written in tensor form as:

$$
\frac{\partial \rho}{\partial t}+\frac{\partial}{\partial x_{i}}\left(\rho u_{i}\right)=0
$$

Conservation of momentum is written:

$$
\frac{\partial}{\partial t}\left(\rho u_{i}\right)+\frac{\partial}{\partial x_{j}}\left(\rho u_{i} u_{j}\right)=-\frac{\partial P}{\partial x_{i}}+\frac{\partial \tau_{i j}}{\partial x_{j}}
$$

Conservation of energy is expressed as follows:

$$
\frac{\partial E_{t}}{\partial t}+\frac{\partial}{\partial x_{j}}\left(u_{j}\left(E_{t}+P\right)\right)=\frac{\partial}{\partial x_{j}}\left(u_{i} \tau_{i j}\right)-\frac{\partial q_{j}}{\partial x_{j}}
$$

Here, the variable $E_{f}$ represents the total energy (internal energy plus kinetic energy) per unit volume:

$$
E_{t}=\rho e+\frac{1}{2} \rho u_{i} u_{i}
$$

The equation of state for an ideal gas is used to relate the pressure, temperature, and density through:

$$
P=\rho R T
$$

For the viscous stresses $\tau_{i j}$, it is assumed that the fluid is a Newtonian fluid, and as a result, the viscous stress is proportional to the rate of strain. This is written:

$$
\tau_{i j}=2 \mu S_{i j}+\lambda \frac{\partial u_{j}}{\partial x_{j}} \delta_{i j}
$$

where the rate of strain tensor $S_{i j}$ is:

$$
S_{i j}=\frac{1}{2}\left(\frac{\partial u_{i}}{\partial x_{j}}+\frac{\partial u_{j}}{\partial x_{i}}\right)
$$

Using Stokes's assumption that the thermodynamic and mechanical pressures are the same for a fluid undergoing and expansion or compression:

$$
\lambda=-\frac{2}{3} \mu
$$

Equation (6) can then be rewritten as:

$$
\tau_{i j}=2 \mu S_{i j}-\frac{2}{3} \mu \frac{\partial u_{j}}{\partial x_{j}} \delta_{i j}
$$

The Sutherland model is used to calculate the viscosity. The heat flux $q_{j}$ is obtained from Fourier's law:

$$
q_{j}=-k \frac{\partial T}{\partial x_{j}}
$$

where $k$ is the thermal conductivity. It is assumed that the fluid is thermally perfect, such that the internal energy and enthalpy are only functions of the temperature, and it is also assumed that the fluid is calorically perfect such that the specific heats $C_{V}$ and $C_{P}$ are constants. As a result, the internal energy and enthalpy can be written as:

$$
\begin{aligned}
e & =C_{V} T \\
h & =C_{P} T
\end{aligned}
$$

Assuming that the air is of constant composition and does not undergo any chemical reaction, the thermal 
conductivity is only a function of temperature. Using the specification of constant specific heats, the following expression is obtained for the thermal conductivity as a function of the constant pressure specific heat. Prandt l number, and the viscosity:

$$
k=\frac{\mu C_{P}}{P r}
$$

\section{Mass-Weighted RANS Equations}

In the classical form of Reynolds averaging, the time dependent form of the Navier-Stokes equations given by (1) through (3) are averaged over a period of time that is much larger than the period of turbulent fluctuations. Each of the dependent variables appearing in these equations is replaced by the sum of mean and fluctuating components. As an example, the velocity would be given by:

$$
u_{i}=\bar{u}_{i}+u_{i}^{\prime}
$$

where the time averaged velocity $\bar{u}_{i}$ is given by:

$$
\bar{u}_{i}=\frac{1}{T} \int_{t}^{t+T} u_{i} d t
$$

For the current work, where fluctuations in density are important, a mass (or density) weighting is employed in the averaging process, which makes the final form of the RANS equations much more convenient to work with. The dependent variables are again broken into mean and fluctuating components:

$$
u_{i}=u_{i}+u_{i}^{\prime \prime}
$$

where the time averaged (using mass weighting) velocity $u_{i}$ is given by:

$$
\dot{u}_{i}=\frac{1}{\bar{\rho} T} \int_{t}^{t+T} \rho u_{i} d t
$$

This mass-weighted Reynolds averaging process is frequently referred to as Favre averaging, and in general, the Favre average of any variable $f$ is defined by:

$$
f=\frac{\overline{\rho f}}{\bar{\rho}}
$$

Applying this averaging procedure to equations (1) (3) results in the following expressions for continuity, momentum, and energy:

$$
\begin{gathered}
\frac{\partial \bar{\rho}}{\partial t}+\frac{\partial}{\partial x_{i}}\left(\bar{\rho} u_{i}\right)=0 \\
\frac{\partial}{\partial t}\left(\bar{\rho} u_{i}\right)+\frac{\partial}{\partial x_{j}}\left(\bar{\rho} u_{i} u_{j}\right)+\frac{\partial \bar{P}}{\partial x_{i}}-\frac{\partial \bar{\tau}_{i j}}{\partial x_{j}}-\frac{\partial \tau_{i j}^{T}}{\partial x_{j}}=0 \\
\frac{\partial}{\partial t}\left(\hat{E}_{t}\right)+\frac{\partial}{\partial x_{j}}\left(u_{j} E_{t}+u_{j} \bar{P}\right)-\frac{\partial}{\partial x_{j}}\left(u_{i} \bar{\tau}_{i j}+u_{i} \tau_{i j}^{T}\right) \\
+\frac{\partial}{\partial x_{j}}\left(\bar{q}_{j}+q_{j}^{T}\right)=0
\end{gathered}
$$

\section{Spatially Filtered LES Equations}

To derive the LES equations used in this work, the time dependent form of the Vavier-Stokes equations given in equations (1) through (3) is again used as the starting point. Instead of time averaging these equations, however, an approach similar to the work of Ragab and Sheen ${ }^{6,7}$ and Erlebacher et al ${ }^{20}$ is used that will filter out small scale fluctuations, and only retain scales that are large enough to be resolved by a particular computational scheme and the computational mesh. The filtering operation is defined on any variable $f$ by the expression:

$$
\bar{f}(\boldsymbol{x}, t)=\int_{D} G(\boldsymbol{x}-\boldsymbol{\xi}, \Delta) f(\boldsymbol{\xi}, t) d^{3} \boldsymbol{\xi}
$$

In equation (21). $G$ is the filter function, $D$ is the flow domain, and $\Delta$ is the filter width. The filter width $\Delta$ is usually taken to be the grid spacing, and is the approach taken here. Note that the overbar used in equation (21) indicates a filtered variable. This is in contrast to the previous use of an overbar to indicate a time averaged quantity in the previous section. As discussed by Nelson. ${ }^{21}$ the exact form of the filter function is not typically known. However, the filter function must satisfy:

$$
\int_{D} G(\boldsymbol{x}-\boldsymbol{\xi}, \Delta) d^{3} \boldsymbol{\xi}=1
$$

In large eddy simulations of compressible flows, it is common to use Favre-filtering which is defined as:

$$
\tilde{f}=\frac{\overline{\rho f}}{\bar{\rho}}
$$

where a quantity $f$ is decomposed into resolved and unresolved (also referred to as sub-grid scale) components as:

$$
f=\tilde{f}+f^{\prime}
$$

Equations (23) and (17) are very similar in appearance, but they refer to very different operations. Both operations employ mass (density) weighting, but equation (17) defines a time averaged quantity, while equation (23) defines a spatially filtered quantity. As was the case for the RANS equations, the density, pressure, and heat flux terms are not decomposed using massweighting. Again note that the overline represented a time averaging process in the previous section, but it will refer to a spatial filtering operation in the current section. In addition, Favre filtering differs from Favre time averaging in that:

$$
\begin{gathered}
\tilde{\tilde{f}} \neq \tilde{f} \\
\tilde{f}^{\prime} \neq 0
\end{gathered}
$$

and 
After applying the filtering procedure to equations (1) - (3) the resulting LES expressions for continuity, momentum, and energy are:

$$
\begin{gathered}
\frac{\partial \bar{\rho}}{\partial t}+\frac{\partial}{\partial x_{i}}\left(\bar{\rho} \tilde{u}_{i}\right)=0 \\
\frac{\partial}{\partial t}\left(\bar{\rho} \tilde{u}_{i}\right)+\frac{\partial}{\partial x_{j}}\left(\bar{\rho} \tilde{u}_{i} \tilde{u}_{j}\right)+\frac{\partial \bar{P}}{\partial x_{i}}-\frac{\partial \bar{\tau}_{i j}}{\partial x_{j}}-\frac{\partial \tau_{i j}^{s g s}}{\partial x_{j}}=0 \\
\frac{\partial}{\partial t}\left(\tilde{E}_{t}\right)+\frac{\partial}{\partial x_{j}}\left(\tilde{u}_{j} \tilde{E}_{t}+\tilde{u}_{j} \bar{P}\right)-\frac{\partial}{\partial x_{j}}\left(\tilde{u}_{i} \bar{\tau}_{i j}+\tilde{u}_{i} \tau_{i j}^{s g s}\right) \\
+\frac{\partial}{\partial x_{j}}\left(\bar{q}_{j}+q_{j}^{s g s}\right)=0
\end{gathered}
$$

\section{Turbulence Modeling}

Both the RANS and LES sets of equations require a turbulence model to close the momentum and energy equations. In the RANS approach, all unsteady turbulent motion is replaced by a turbulence model. The resulting LES equations are very similar in appearance to the RANS equations, and also require a model to close the momentum and energy equations. The difference for the LES equations, however, is that the terms replaced by a model are only the turbulent terms that are too small to be resolved using the filtered LES equations. As a result, the large scale turbulent motion is directly calculated, and the effects of the smallest scale turbulence are accounted for using a subgrid turbulence model.

The turbulence model employed here to close the RANS equations is the Cebeci-Smith algebraic turbulence model. ${ }^{22,23}$ Since the RANS equations are only used in this hybrid method to calculate wall boundary layer regions with no adverse pressure gradients, the selection of a relatively simple algebraic model such as the Cebeci-Smith formulation is appropriate. The wall function technique of Ota and Goldberg ${ }^{24}$ is used in conjunction with the Cebeci-Smith model to enable use of a computational grid with the first point off solid boundaries placed in the logarithmic layer. This wall function approach is based upon the compressible law of the wall formulation of White and Christoph. ${ }^{25,26}$ The filtered LES equations are closed using the Smagorinsky subgrid model. ${ }^{27}$

Implementation of the wall function technique is critical to the development of this hybrid approach in order to enable use of a single computational grid extending continuously from the RANS regions to the LES regions. If a wall function approach were not used, grids for the RANS regions would have to be packed very tightly to the wall and use significant grid stretching, while a separate grid which minimizes grid stretching would need to be constructed for the LES region. Use of such non-continuous grids for the RANS and LES regions would require a scheme that would likely introduce interpolation errors into the combined hybrid method.

Use of the Cebeci-Smith model to close the RANS equations and the Smagorinsky subgrid model to close the LES equations is desirable in terms of code implementation. While the function of the Cebeci-Smith model to replace all of the turbulent stresses with a model is quite different from that of the Smagorinsky subgrid model, which only replaces the small subgrid turbulent stresses, both are eddy viscosity models and are derived at least in part from mixing-length theory. The similar formulation of these two models enables the RANS equations and LES equations to be solved with a single solution scheme and computational grid, as mentioned previously. For a compressible nozzle or mixing layer flow, such as that depicted in figure 1 , the change from RANS regions to LES region occurs at the vertical plane passing through the trailing edge of the splitter separating the wall bounded flows.

\section{RANS Turbulence Model}

The unclosed terms from the RANS momentum and energy equations are the Reynolds stress and turbulent heat flux. The Boussinesq approximation is used to relate the turbulent Reynolds stress to the mean rate of strain tensor through a turbulent (or eddy) viscosity. This is directly analogous to equation (9) which relates the viscous stress to the mean rate of strain through the molecular (or laminar) viscosity. The turbulent analogy to equation (9) is:

$$
\begin{aligned}
\tau_{i j}^{T} & =-\overline{\rho u_{i}^{\prime \prime} u_{j}^{\prime \prime}} \\
& =\mu^{T}\left(2 \dot{S}_{i j}-\frac{2}{3} \frac{\partial \hat{u}_{k}}{\partial x_{k}} \delta_{i j}\right)
\end{aligned}
$$

Similarly, the turbulent heat flux is related to the temperature gradient through a turbulent conductivity. $k^{T}$ :

$$
\begin{aligned}
q_{j}^{T} & =\overline{\rho u_{j}^{\prime \prime} h^{\prime \prime}} \\
& =-k^{T} \frac{\partial T}{\partial x_{j}}
\end{aligned}
$$

The turbulent Prandtl number, $P r^{T}$ is used to relate the turbulent viscosity to the turbulent conductivity:

$$
P r^{T}=\frac{\mu^{T} C_{P}}{k^{T}}
$$

The turbulent Prandtl number is taken to be a constant here and equal to 0.9 . Using equation (32) and assuming a constant turbulent Prandtl number enables the turbulent heat flux to be expressed as a function of the turbulent viscosity that is used to calculate the Reynolds stress. The turbulent heat flux becomes:

$$
q_{j}^{T}=-\frac{C_{P} \mu^{T}}{P r^{T}} \frac{\partial \hat{T}}{\partial x_{j}}
$$


The Cebeci-Smith model, which was chosen here to close the RANS equations in the wall boundary layer regions, treats the wall boundary layer as having inner and outer regions where the turbulent viscosity is defined as:

$$
\mu^{T}= \begin{cases}\mu_{1 \text { ner }}^{T}, & y \leq y_{m} \\ \mu_{\text {rater. }}^{T}, & y>y_{m}\end{cases}
$$

In equation (34), $y_{m}$ is defined as the smallest value of $y$ (the distance away from a wall) at which $\mu_{\text {inner }}^{T}=$

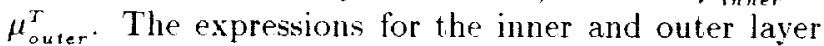
turbulent viscosities are as follows:

Inner Layer:

$$
\mu_{\text {inner }}^{T}=\bar{\rho} \epsilon_{m i x}^{2} \sqrt{\left(\frac{\partial u}{\partial y}\right)^{2}+\left(\frac{\partial u}{\partial x}\right)^{2}}
$$

with the mixing length $\epsilon_{m x}$ is given by:

$$
\ell_{m x x}=\kappa y\left(1-e^{-y^{+} / A^{+}}\right)
$$

Note that the specific form of equation (35) is for a two-dimensional boundary layer.

Outer Layer:

$$
\mu_{\text {outer }}^{T}=\alpha \bar{\rho} \delta_{i} u_{\epsilon} F_{k l e b}
$$

In equation $(37)$, the quantity $\delta_{v}$ is the velocity thickness, $u_{e}$ is the boundary layer edge velocity, and $F_{k l e b}$ is the Klebanoff intermittency function. The velocity thickness is defined as:

$$
\delta_{v}=\int_{0}^{\delta}\left(1-\frac{u}{u_{e}}\right) d y
$$

This velocity thickness is identical to the displacement thickness for incompressible flows. Klebanoff ${ }^{28,29}$ presented an expression for the intermittency of turbulence near the edge of a boundary, which has a functional form involving the complimentary error function. This original intermittency function is usually approximated by the following formula, as indicated by Cebeci: ${ }^{22}$

$$
F_{k l e b}=\left[1+5.5\left(\frac{y}{\delta}\right)^{6}\right]^{-1}
$$

The closure coefficients appearing in equations (36) and $(3 i)$ are

$$
\kappa=0.40 \quad \alpha=0.0168 \quad A^{+}=26
$$

Wall Function Implementation: The Cebeci-Smith model is usually integrated down to the wall, using a computational grid with the first point off of the wall placed well within the laminar sublayer, corresponding to $y^{+}<5$. For the hybrid method developed in this work, the objective is to place the first point off of the wall in the logarithmic layer to enable the use of computational grids that are not packed as tightly to the wall. Removing the tight spacing requirement will enable a continuous grid into the LES region. In addition, because the allowable time step of the computations is directly proportional to the size of the smallest grid cell, a less tightly parked grid enables a larger time step for the solution scheme. "The' wall function technique of $\mathrm{O} 1 \mathrm{a}$ and Coldberg ${ }^{24}$ is one of the more simple and effective methods currently in use, and it is the technique used in this work.

Wall functions have been implemented most frequently in conjunction with two-equation $k-\epsilon$ models. The benefits of implementing a wall function for use with a $k-f$ model are the same as that for the CebeciSmith model used in this work including reducing grid requirements, and increasing the permissible time step of the computations.

The use of a wall-function approach is strictly only valid in flow regions absent of adverse pressure gradients and separations, due to the assumption that the law of the wall holds. However, Avva et al. ${ }^{30}$ have shown results for separated flows in which wall function methods perform no worse than methods integrating to the wall. The intention of the wall function implementation in this work is to only apply the method to attached wall boundary layers where the law of the wall is valid.

The Ota-Goldberg wall function employs the WhiteChristoph ${ }^{25,26}$ compressible law of the wall:

$$
u_{2}^{+}=\frac{1}{\sqrt{\gamma}} \sin \left(\frac{\sqrt{\gamma}}{\kappa} \ln \frac{y_{2}^{+}}{y_{0}^{+}}\right)
$$

where the compressibility parameter $\gamma$ is given by:

$$
\gamma=\frac{r u_{\tau}^{2}}{2 C_{P} T_{u}}
$$

In equation (40) $u_{2}^{+}$is the value of $u^{+}$at the first point off of the wall, $y_{2}^{+}$is the value of $y^{+}$at the first point off of the wall, and $y_{0}^{+}=0.1287$. In equation (41), the parameter $\mathrm{r}$ is the recovery factor, which is typically taken to be $\operatorname{Pr} \frac{1}{3}$ for turbulent boundary layers, and $T_{u}$ is the wall temperature. An iteration procedure is used with equations (40) and (41) to solve for $u_{2}^{+}$, from which the shear velocity $u_{\tau}$ can be obtained:

$$
u_{2}^{+}=\frac{u_{2}}{u_{\tau}}
$$

Finally, the shear velocity is used to compute the wall shear stress through:

$$
\tau_{u}=\rho u_{\tau}^{2}
$$

The wall shear stress calculated in equation (43) is then used in the solution scheme for the momentum and energy equations in the RANS regions. 


\section{LES Subgrid Scale Model}

The terms that must be closed for LES equations are the subgrid-scale stress and the subgrid scale heat flux. The earliest subgrid scale model for LES computations was developed by Smagorinsky. ${ }^{27}$ Despite significant efforts to develop more sophisticated subgrid scale models, the Smagorinsky formulation is still widely used, and is itself the foundation upon which some of the more sophisticated models are derived. The form of the model is very similar to the CebeciSmith model used for the RANS equations, in that a gradient-diffusion mixing-length approach is used.

The Snagorinsky expression for the subgrid scale stress is:

$$
\begin{aligned}
\tau_{i j}^{s g s} & =\bar{\rho}\left(\widetilde{u_{i} u_{j}}-\tilde{u}_{i} \tilde{u}_{j}\right) \\
& =2\left(C_{S} \Delta\right)^{2} \bar{\rho} \sqrt{\pi}\left(\tilde{S}_{i j}-\frac{1}{3} \tilde{S}_{k k} \delta_{i j}\right)-\frac{2}{3} C_{I} \Delta^{2} \bar{\rho} \pi \delta_{i j}
\end{aligned}
$$

The parameter $\pi$ is defined:

$$
\pi=\tilde{S}_{i j} \tilde{S}_{i j}
$$

The parameter $\Delta$ is the filter width and as a result, it is also used as the length scale that is characteristic of the subgrid turbulence. For use with a computational method, $\Delta$ is usually taken to be the grid spacing. In three dimensions, with a computational grid having unequal spacing in the three directions, this subgrid length scale is usually taken to be:

$$
\Delta=(\Delta x \Delta y \Delta z)^{\frac{1}{3}}
$$

For computational grids with substantially different spacing in the three directions, an alternative form (see Ragab $^{7}$ ) is

$$
\Delta=\left[\frac{(\Delta x)^{2}+(\Delta y)^{2}+(\Delta z)^{2}}{3}\right]^{\frac{1}{2}}
$$

This second expression for the length scale is employed in this work. The constants $C_{S}$ and $C_{I}$ have been found to be dependent on the flow under investigation. Rogallo and $\mathrm{Main}^{31}$ suggest a range for $C_{S}$ in the range $0.10 \leq C_{S} \leq 0.24$. The upper limit on $C_{S}$ given by Rogallo and Moin is investigated for the mixing layer in this work. The constant $C_{I}$ is usually equal to 0.01 , but several authors, including $\mathrm{Ragab}^{\top}$ and Choi et al ${ }^{32}$ mention that the contribution of the term involving $C_{I}$ may not be important and may be neglected. This approach is taken in this work, and as a result, the original expression for the Smagorinsky subgrid scale stress in equation (44) may be rewritten as follows:

$$
\tau_{i j}^{s g s}=2 \mu^{s g s} \tilde{S}_{i j}-\frac{2}{3} \mu^{s g s} \frac{\partial \tilde{u}_{j}}{\partial x_{i}} \delta_{i j}
$$

where the subgrid scale turbulent viscosity is given by:

$$
\mu^{s g s}=\bar{\rho}\left(C_{S} \Delta\right)^{2} \sqrt{\pi}
$$

Note the similar form of equation (49) to the expression for the Cebeci-Smith inner region turbulent viscosity in equation (35). While the mixing length defined for the Cebeci-Smith model is used to characterize all of the turbulent motion, the length scale defined here for the Smagorinsky model only characterizes the subgrid-scale motion. Finally, the subgrid scale heat flux is modeled analogously to that done for the turbulent heat flux of the RANS equations:

$$
\begin{aligned}
q_{j}^{\mathrm{sg}} & =\bar{\rho}\left(\overline{u_{j} h}-\tilde{u}_{j} \tilde{h}\right) \\
& =-k^{s g s} \frac{\partial \dot{T}}{\partial x_{j}}
\end{aligned}
$$

where $k^{s q s}$ is related to $\mu^{s s^{s}}$ through the turbulent Prandtl number. As in the RANS regions, the turbulent Prandtl number is assumed to be constant in the LES regions and equal to 0.9 . The subgrid scale heat flux becomes

$$
q_{j}^{s g s}=-\frac{C_{P} \mu^{s g s}}{P r^{T}} \frac{\partial \hat{T}}{\partial x_{j}}
$$

\section{Solution Procedure}

The similar form of the RANS and LES equations derived here enable both sets to be solved with a single computational method. In this work, the GottliebTurkel scheme ${ }^{33}$ was used. In addition, the equations are transformed to generalized coordinates and solved using a set of metric terms that are consistent with the order of the Gottlieb-Turkel scheme. This was done to enable the use of the hybrid method on stretched, non-Cartesian grids. The Gottlieb-Turkel scheme is cited by several authors, such as Hudson and Long, ${ }^{34}$ as providing second order accuracy in time and fourth order accuracy in space. Bayliss et al. ${ }^{35}$ indicated that the scheme has fourth order accuracy only if $\Delta t$ is of the order $(\Delta x)^{2}$, and Nelson ${ }^{21}$ showed that the spatial accuracy of the scheme is strictly only third order for CFL numbers approaching 1 . In the case of simulations with grids employing significant stretching, however, the spatial accuracy is effectively fourth order for most of the computational domain.

\section{Mixing Layer Simulations}

\section{Experimental Configuration}

The hybrid RANS/LES method has been applied to one of the benchmark compressible mixing layer experiments of Goebel and Dutton ${ }^{36-38}$ in which two isolated supersonic streams, separated by a splitter plate, provide the flows to a constant area mixing section. In particular, case 2 of their experiments is examined here. A simplified schematic of their experimental configuration provided in figure 2 shows 


\begin{tabular}{ccc} 
& top flow & bottom flow \\
\hline MachNo. & 1.91 & 1.36 \\
T(m/s) & 700 & 399 \\
$T_{t}(\mathrm{~h})$ & 578 & 295 \\
$T(\mathrm{~K})$ & 334 & 215 \\
$a(\mathrm{~m} / \mathrm{s})$ & 366 & 293 \\
$P(\mathrm{kPa})$ & 49 & 49 \\
$\rho\left(\mathrm{kg} / \mathrm{m}^{3}\right)$ & 0.51 & 0.79 \\
$\delta(\mathrm{mm})$ & 2.9 & 2.5 \\
$\delta^{*}(\mathrm{~mm})$ & 0.90 & 0.44 \\
$\theta(\mathrm{mm})$ & 0.29 & 0.21
\end{tabular}

Table 1 Flow conditions for case 2 of the GoebelDutton experiments

that two isolated streams, in which boundary layers develop over a splitter plate surface, are brought together into a constant area mixing section. In all of their experiments, the higher speed primary stream occurred over the top surface of the splitter plate. The top stream enters the mixing section axially, while the bottom stream enters the mixing section at an angle of 2.5 degrees. The splitter plate thickness has a base height of $0.5 \mathrm{~mm}$ at the trailing edge. Upstream of the straight sections for the two isolated flows shown in figure 2, contoured nozzle blocks provided the supersonic flows with nearly uniform exit flow conditions.

The mixing section height was $48 \mathrm{~mm}$, and the overall length of the mixing section available for flowfield measurements was $500 \mathrm{~mm}$. The width of the mixing section was $96 \mathrm{~mm}$, and as a result, the mean flow development could be considered two-dimensional. The divergence angle of the lower and upper walls of the mixing section were adjusted in each experiment with two incoming supersonic flows, to account for boundary layer growth along these two surfaces and to effectively remove any streamwise pressure gradient.

Single components LDV measurements were used to calculate the boundary layer, displacement, and momentum thicknesses of the two streams as they entered the mixing section. These quantities are provided along with the other operating conditions of case 2 in table 1. The documentation of the incoming boundary layer characteristics makes the Goebel-Dutton experiments one of the more thoroughly documented benchmark data sets available for compressible mixing layers. In the mixing region, a two-component LDV system was used to measure the axial and transverse velocities. In addition, a Schlieren system with a 20 ns pulse duration was used to obtain nearly instantaneous snapshots of the mixing layer.

\section{RANS Validation}

To begin the investigation of the hybrid method, initial RANS calculations for the two streams feeding the mixing layer were constructed. For the Mach 1.91 stream, wall-integration and wall-function grids were generated. Both grids extended $300 \mathrm{~mm}$ axially and
$150 \mathrm{~mm}$ vertically. In addition, both grids used 141 points in the axial direction and 141 points in the vertical direction. The wall-integration grid was packed to the wall such that the first grid spacing was 0.006 $m m$, corresponding to an average $y^{+}$of 2.5 . The wall function grid had the first point placed at $0.05 \mathrm{~mm}$. corresponding to an average $y^{+}$of approximately 20 This wall spacing was chosen for use with the wallfunction grid so that the initial grid spacing at the wall was exactly $1 / 10$ th of the splitter plate thickness in the experiment. This grid spacing could then be continued into the mixing region, with 10 points spaced equally in the vertical direction at the base of the splitter. In the axial direction, both the wall-integration and wall-function grids were packed to leading and trailing edges with spacings at the two ends set to $0.10 \mathrm{~mm}$. corresponding $101 / 5$ th of the splitter base thickness.

For the Mach 1.36 case. grid construction was very similar to that used for the Mach 1.91 stream, with the exception that the axial dimension was shorter for the Mach 1.36 calculations. This was done because the Mach 1.36 boundary layer thickness in the DuttonGoebel experiment measured at the beginning of the mixing section was smaller than the Mach 1.91 boundary layer. The grids extended $200 \mathrm{~mm}$ in the axial direction and $150 \mathrm{~mm}$ vertically. Both grids used 141 points in the axial and vertical directions. The wallintegration grid was packed such that the first spacing was $0.006 \mathrm{~mm}$, corresponding to an average $y^{+}$of approximately 3.0. The wall function grid placed the first point at $0.05 \mathrm{~mm}$, corresponding to an average $y^{+}$of approximately 25.

For each of the cases discussed in this section, the wall boundary condition for the calculations was set to be an adiabatic no-slip surface and the extreme vertical boundary was set as a slip surface. The inflow boundary was fixed to uniform supersonic flow and the outflow boundary extrapolated all quantities.

Figure $3(\mathrm{a})$ compares velocity profiles obtained with the two approaches to analytical predictions from the compressible boundary layer method of Tucker ${ }^{39}$ for the Mach 1.36 boundary layer. Figure 3(b) provides the same comparison for the Mach 1.91 boundary laver. The solutions provided with and without the wall-function method provide close agreement with the Tucker theory. Figure 4 provides a comparison of the momentum thickness growth along the two flat plates and also indicates close agreement between the calculations and the method of Tucker. These results verify that the RANS part of the current method accurately predicts the mean flow behavior of the supersonic boundary layers entering the mixing section.

\section{Two-Dimensional Mixing Layer Calculations}

While true LES simulations require computations in three spatial directions, initial two-dimensional hybrid calculations of the entire mixing layer configuration 


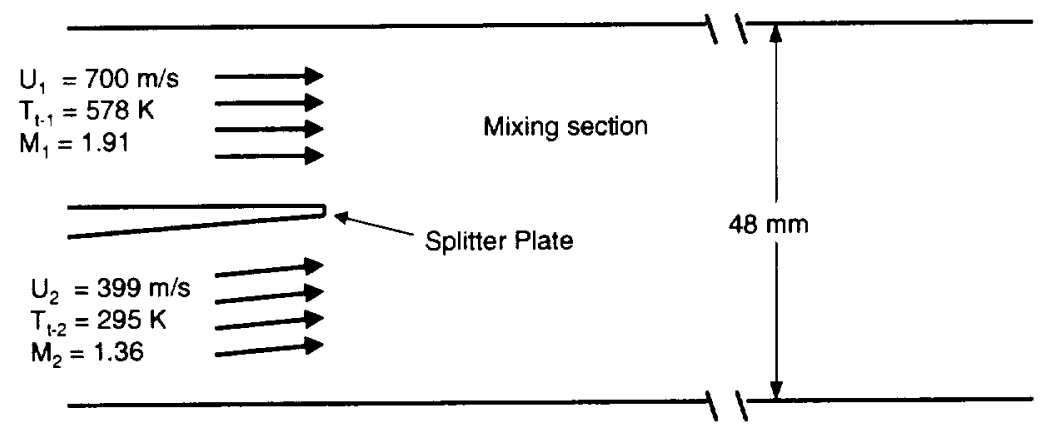

Splitter Plate Parameters:

T.E. thickness $=0.50 \mathrm{~mm}$

not to scale

Bottom surface angle $=2.5^{\circ}$

Fig. 2 Schematic of Goebel-Dutton mixing layer experiment and Case 2 operating conditions

were used to investigate effects of axial grid resolution and the splitter plate treatment. No subgrid model was used in these preliminary calculations.

The development of the computational model began by using the results of the Mach 1.91 and Mach 1.36 boundary layer simulations. Specifically, the wallfunction solutions were used, because the computational grids employed with the wall-function approach enabled a continuous grid into the mixing region for use with the hybrid RANS-LES solver. The objective was to construct two RANS regions that would provide boundary layer quantities $\delta, \delta^{*}$, and $\theta$ that nearly matched those measured in the experiment. Because it would be virtually impossible to match all of three quantities exactly, the momentum thickness $\theta$ was chosen as the key parameter to match the computations with the experiment. The momentum thickness represents the mean momentum deficit entering the mixing section and is fundamental to the mixing layer behavior.

Examining the Mach 1.91 boundary layer first, table 1 indicates that the momentum thickness for this stream was measured as $0.29 \mathrm{~mm}$ at the trailing edge of the splitter plate. For the wall function calculations discussed in the previous section, the momentum thickness reached $0.29 \mathrm{~mm}$ at a Reynolds number of $3,680,000$ (see figure $4(\mathrm{~b})$ ), corresponding to an axial position of $198 \mathrm{~mm}$ from the leading edge of the plate.

The next step was to construct a new computational grid. The axial domain was shortened to $198 \mathrm{~mm}$ while retaining the same number (141) of axial grid points. The grid stretching was modified to accommodate the shorter domain while maintaining the initial and ter- minal grid spacings. The vertical domain was reduced to $23.75 \mathrm{~mm}$ to exactly match the height of the Mach 1.91 stream in the experiment. The vertical domain of the original grid reached $23.75 \mathrm{~mm}$ at the $94 \mathrm{th}$ grid point, so the first 94 points from the original grid were used in the modified grid. Calculations obtained with this $141 \mathrm{x} 94$ point, $198 \mathrm{~mm}$ by $23.75 \mathrm{~mm}$ grid provided boundary layer quantities identical to that of the original $141 \times 141$ grid, further validating the grid independent characteristics of the RANS method.

A similar procedure was used for the Mach 1.36 boundary layer. Table 1 indicates that the momentum thickness for this stream was $0.21 \mathrm{~mm}$ at the splitter trailing edge. Examination of the wall-function solution obtained for the Mach 1.36 boundary layer revealed that the momentum thickness became $0.21 \mathrm{~mm}$ at an axial position of approximately $120 \mathrm{~mm}$, corresponding to a plate Reynolds number of $2,720,000$. A modified grid was constructed using 141 axial and 94 vertical points for this Mach 1.36 case, corresponding to a physical domain of $120 \mathrm{~mm}$ by $23.75 \mathrm{~mm}$. Calculations with this grid provided a solution identical to that obtained with the original $141 \times 141$ grid.

The last step before constructing the entire RANSLES computational grid was to extract the grid points from the modified wall-function grids just discussed, extending from 81-141 in the axial domain. The solutions were then held constant at the 8 lst axial station as the inflow of the final hybrid RANS-LES grid. This was done to reduce the grid requirements for the subsequent RANS-LES computations. Boundary layer calculations subsequently obtained with these two shortened axial grids using 61 axial points and 94 vertical 


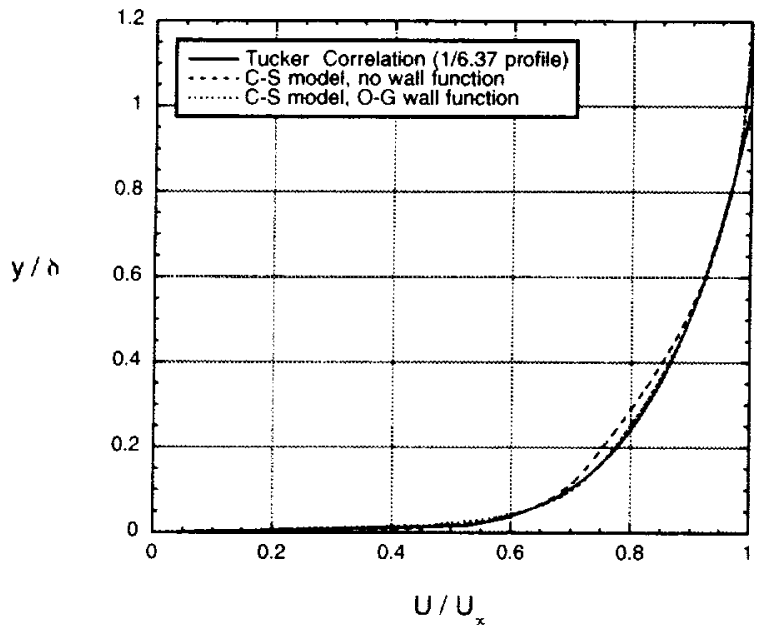

a) Mach 1.36

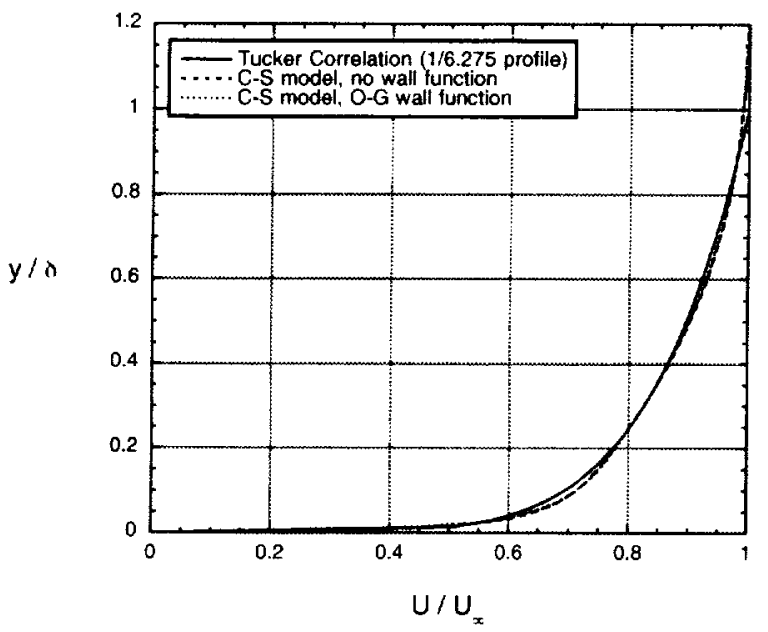

b) Mach 1.91

Fig. 3 Velocity profiles for boundary layer calculations

points again returned the same boundary layer quantities and velocity profiles as the other solutions. These were the RANS grids that were used to join with the LES mixing region computational domain.

Three axial grid spacings (with 200,400, and 800 points in the mixing section respectively) were examined in the initial two-dimensional hybrid calculations. In all cases, however, the vertical spacing from the two RANS regions was continued throughout the entire LES region. With the tightest vertical spacing of the wall-function boundary layer solutions set to 0.05 $\mathrm{mm}, 10$ grid spacings were used vertically across the splitter base. As a result, all of the hybrid grids used 197 vertical points in the mixing region.

Fixed inflow boundary conditions were used at the RANS inflows. The fixed inflow for the Mach 1.91 upper stream was placed at an axial position $67 \mathrm{~mm}$ upstream of the splitter plate trailing edge and the Mach 1.36 lower stream inflow was placed $42 \mathrm{~mm}$ up-

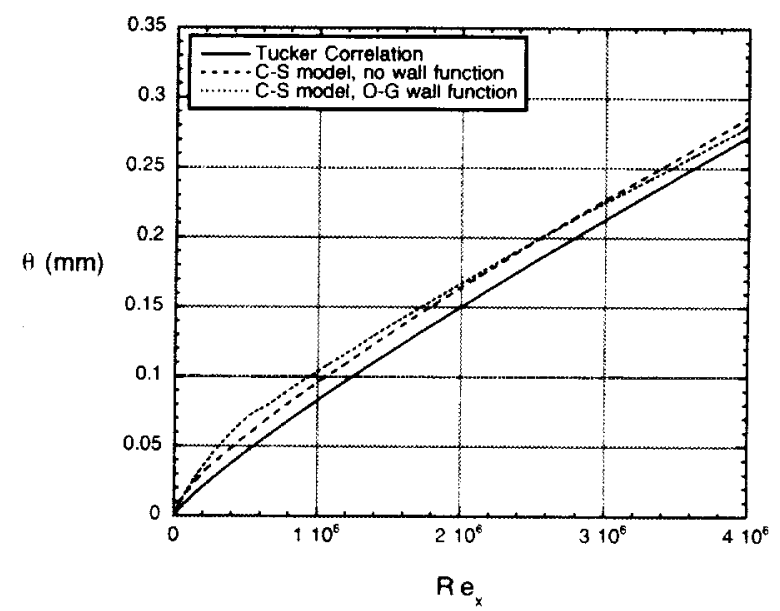

a) Mach 1.36

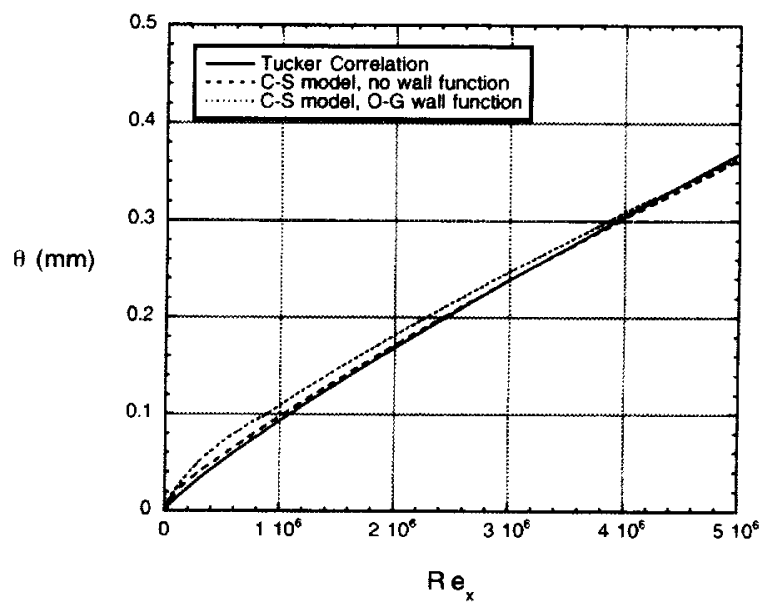

b) Mach 1.91

Fig. 4 Momentum thickness growth along supersonic flat plates

stream of splitter tip. At the outflow of the mixing section, corresponding to an axial distance of $300 \mathrm{~mm}$ from the trailing edge of the splitter plate, an extrapolation boundary condition was used, which is appropriate for the mixing supersonic flow exiting the axial domain. The top and bottom walls of the mixing section were approximated as slip walls, and no attempts were made to simulate boundary layers developing on these two surfaces. The Goebel-Dutton experiment configuration was specifically designed with adjustable divergence angles for these walls to account for boundary layer growth, and to thereby provide data that may be directly compared to calculations which do not include the mixing section boundary layers.

The first two-dimensional simulation investigating grid density effects was for the coarsest grid using 200 axial points. A vortex shedding pattern was observed to originate from the trailing edge of the splitter plate due to the separation of the two flows leaving the wall bounded RANS regions and entering the LES mixing 
section. The vortex shedding quickly dissipates and the flow appeared to be mostly laminar throughout the rest of the mixing section.

The second two-dimensional simulation was for the computational grid using 400 axial points. The characteristics of this flow were substantially different from those of the 200 grid point case. Instantaneous density contours are provided in figure 5. A stronger vortex shedding is evident for this case, although the vortex strength gradually dissipates back to a laminar state approximately $40 \mathrm{~mm}$ downstream of the splitter trailing edge. An instability forms at an axial position of $80 \mathrm{~mm}$ which in turn slightly dissipates before resuming growth at a position $180 \mathrm{~mm}$ downstream of the splitter plate trailing edge. A turbulent pattern then grows from this location to the exit at $x=300 \mathrm{~mm}$.

The 400 point axial grid consisted of smaller axial grid steps than the 200 axial point grid and had a significantly reduced stretching factor relative to the coarse grid. As a result, the truncation error term in the Gottlieb-Turkel scheme that effectively smooths discontinuities is substantially reduced for the $400 \mathrm{ax}$ ial point grid, and the capability to resolve unsteady flow behavior was improved.

The third computational grid investigated had 800 axial points. A substantially different flow behavior was also observed for this case compared to the solutions obtained with 200 and 400 axial points. The density contours in figure 6 again indicate a vortex shedding pattern that originates from the trailing edge of the splitter plate, but unlike the 400 point case, the solution does not return to a laminar state before transitioning over to a turbulent-like pattern at $x=80$ $\mathrm{mm}$. The very tight axial spacing for this case is sufficient to minimize the truncation error damping effects on the unsteady flow development.

An additional two-dimensional grid with 800 axial points was constructed for a modified splitter geometry in which the splitter trailing edge is reduced to a sharp tip, and as a result. the flow separation and vortex shedding is removed. Figure 7 provide instantaneous density contours for the case with a sharp trailing edge. As expected, the vortex shedding evident in the baseline case was removed in the current case with the sharp tip. The lack of a separation region results in an initially laminar mixing layer through the beginning of the mixing section. Interestingly, the laminar flow begins to transition to a more turbulent structure at nearly the same position observed for the baseline case, at approximately $x=80 \mathrm{~mm}$. The structures remain relatively small until $x=150 \mathrm{~mm}$ where large scale turbulence forms. These structures are more similar to the Brown-Roshko organized structures than were those of the baseline case with 800 axial points.

\section{Three-Dimensional Mixing Layer Calculations}

In the previous section, two-dimensional calculations were used to construct the initial computational model of the mixing layer and to examine preliminary effects of grid resolution and splitter plate treatment. To correctly investigate the capability of the hybrid method, however. LES calculations obtained in three spatial directions with the use of a subgrid scale model were performed.

The grid topologies and boundary conditions used for the three-dimensional simulations were very similar to those used for the two-dimensional simulations. The two-dimensional computational grids with 200 . 400 , and 800 axial point grids were used to construct the three-dimensional grids used liere. To add the third computational direction in each case, the two-dimensional planar grid was copied to provide 11 points in the third (or $z$ ) direction. The grid spacing in the $z$ direction was uniform and set equal to the axial spacing at the splitter trailing edge, or $\Delta z=$ $\Delta x_{1}=0.10 \mathrm{~mm}$. Because of the very small number of grid points used in the $z$ direction and the small physical space that is represented, only very small wave components in this direction could be simulated, and a periodic boundary condition was used in this direction.

All of the other boundary conditions and the soJution procedure are identical to that used for the two-dimensional mixing layer calculations, with the one exception being that the Smagorinsky subgrid scale model was used in these three-dimensional simulations. The switch from the RANS regions to the LES region at the mixing plane (corresponding to a vertical plane drawn through the trailing edge of the splitter plate) was accomplished by changing the eddy viscosity used in the flow solver from the Cebeci-Smith turbulence model to the Smagorinsky subgrid scale model. As a result, the effect of the eddy viscosity changes from that of replacing all of the turbulent stresses in the RANS regions to that of only replacing the subgrid stresses in the LES regions.

The first three-dimensional simulation was obtained using the computational grid with 200 axial points. The behavior of the flow just downstream of the splitter trailing edge is very similar to the corresponding two-dimensional calculation. The lack of adequate axial grid resolution results in a rapid dissipation of the initial vortex pattern, and is even more rapid in the three dimensional case due to the dissipative nature of the Smagorinsky subgrid scale model.

The three-dimensional grid using 400 axial points was utilized next. The density contours shown in figure 8 indicate a fundamentally different flow structure than observed with any of the two-dimensional calculations. In particular, the vortex shedding is observed to transition into a turbulent pattern much closer to the trailing edge. While the Schlieren photographs 


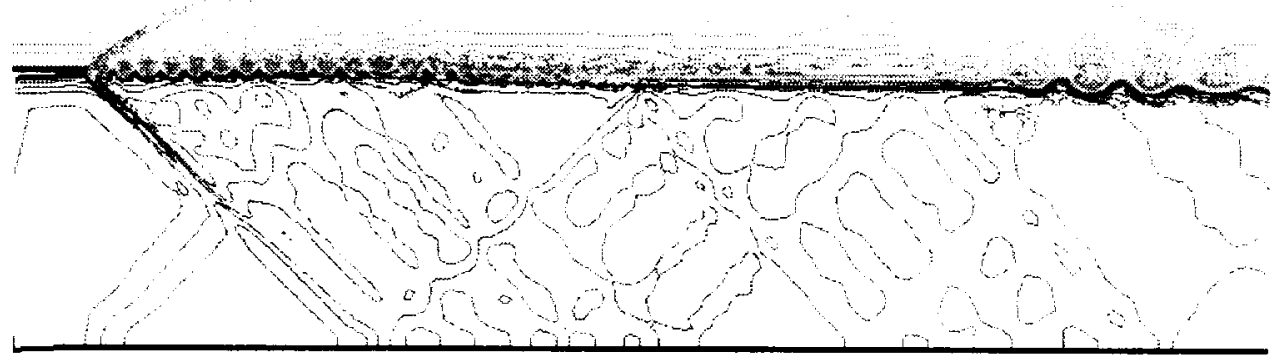

a) Beginning of mixing section

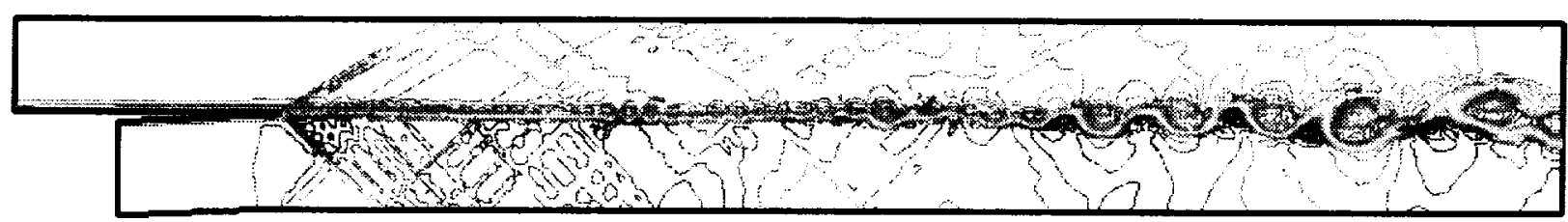

b) Entire mixing section

Fig. 5 Instantaneous density contours for the 400 axial grid point case (2D)

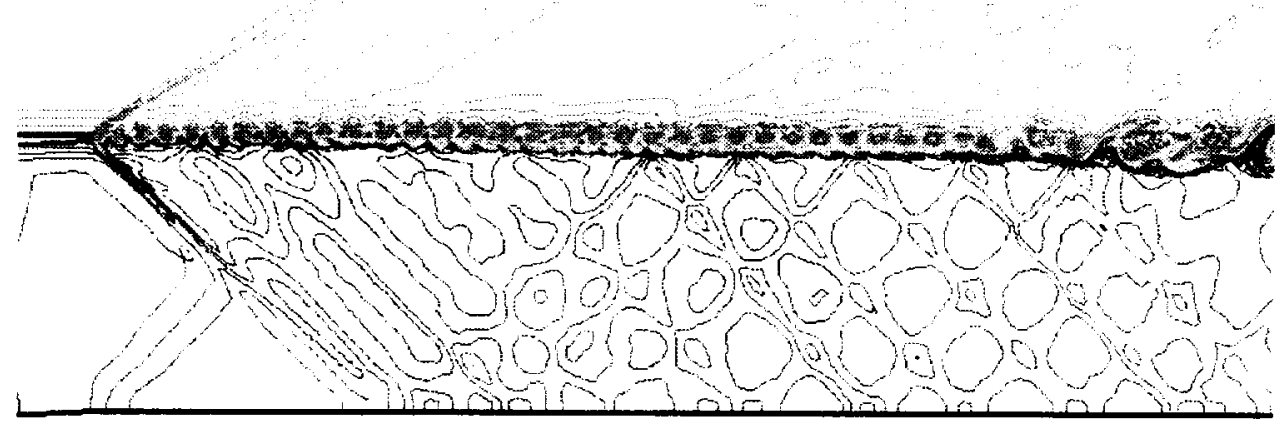

a) Beginning of mixing section

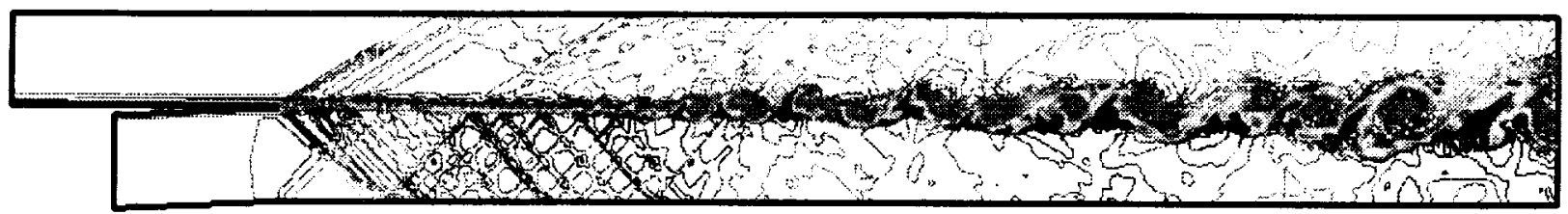

b) Entire mixing section

Fig. 6 Instantaneous density contours for the baseline 800 axial grid point case (2D) 


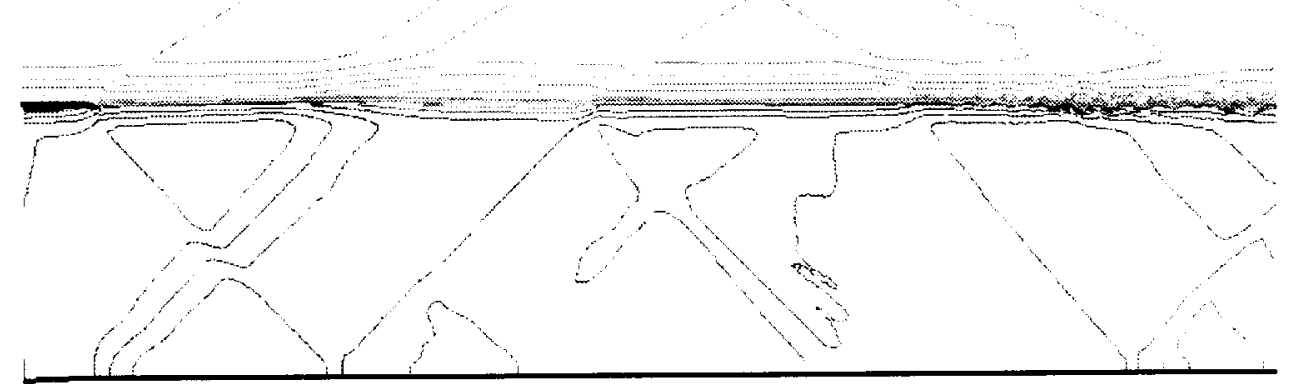

a) Beginning of mixing section

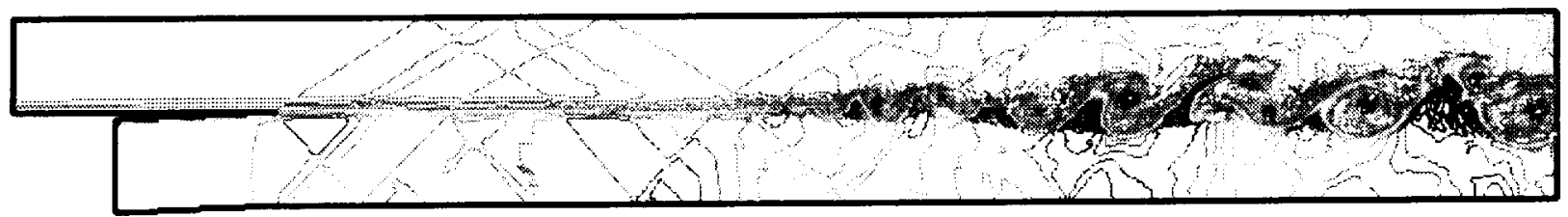

b) Entire mixing section

Fig. 7 Instantaneous density contours for the 800 axial grid point case with a sharp trailing edge for the splitter (2D)

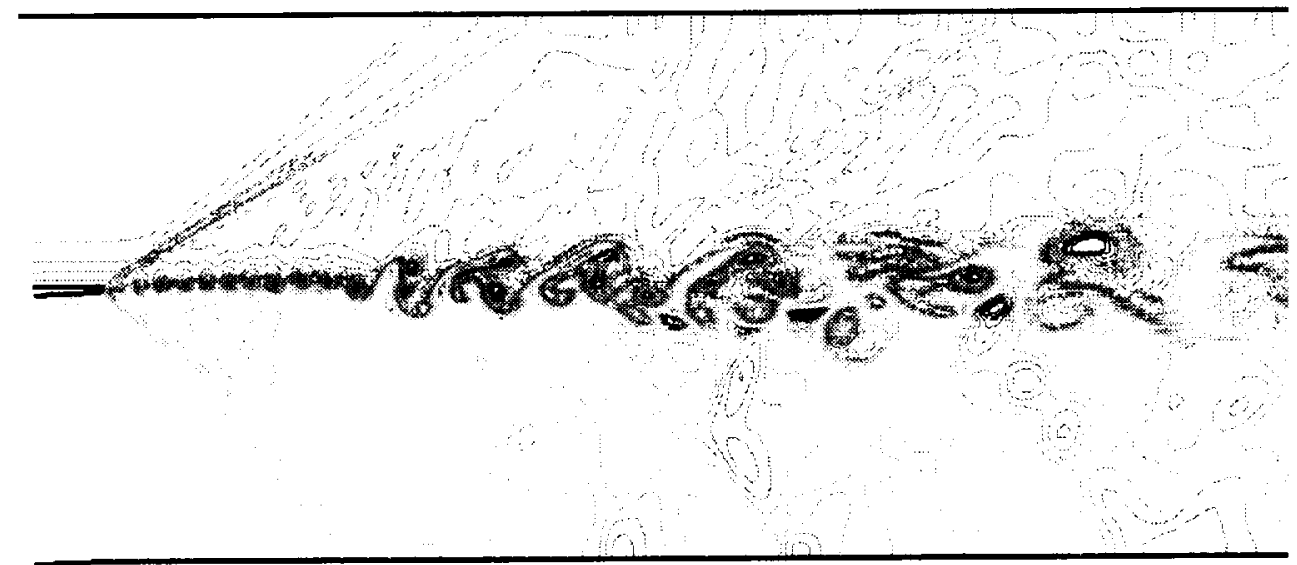

a) Beginning of mixing section

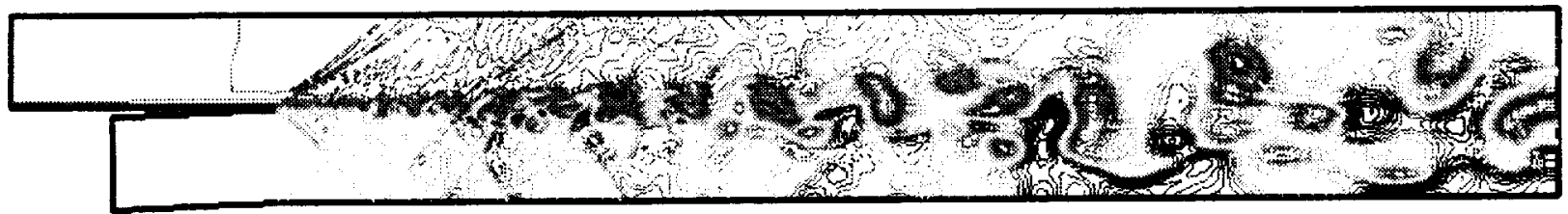

b) Entire mixing section

Fig. 8 Instantaneous density contours for the 3D case using 400 axial points 


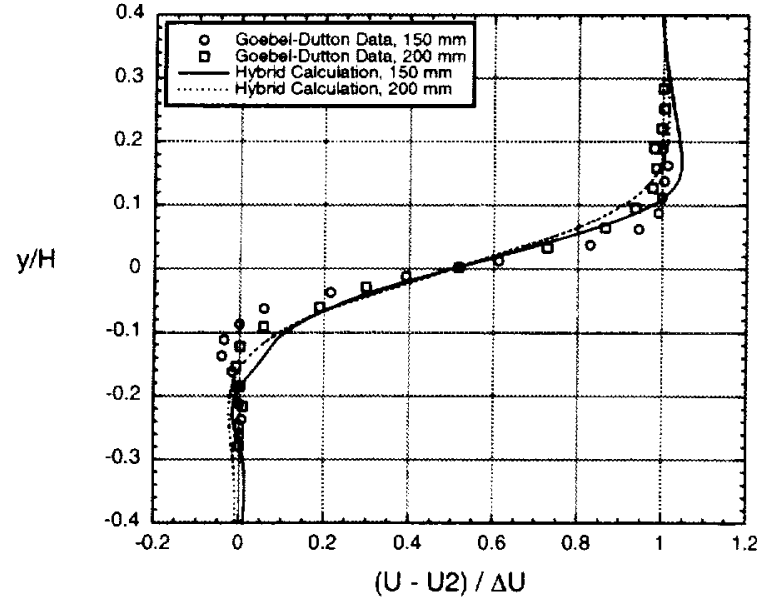

Fig. 9 Velocity profiles in the mixing section

from the Goebel-Dutton experiments did not probe the flow details near the splitter plate trailing edge, such details were examined by Clemens and Mungal ${ }^{40}$ for a similar experiment configuration and flow operating conditions. A Schlieren photograph from the ClemensMungal experiments indicates an initial flow structure similar to that observed in the three-dimensional calculation with initial vortex shedding from the trailing edge of a splitter plate followed by a rapid transition into turbulence. The turbulent structure in the experiment was observed to be of primarily small scales, while the LES calculations, by definition, only capture the large scale structures. While the length of the organized vortex structure in the calculations does not exactly match that of the Clemens-Mungal experiment, the three-dimensional calculations predict the transition location much more accurately than the previous two-dimensional results. In addition, these results verify that LES calculations must be run in three dimensions to allow instabilities to form in all three directions.

Mean axial profiles obtained from this threedimensional calculation are compared to experimental data of Goebel and Dutton in figure 9. The calculation indicates greater shear layer spreading than shown by the experimental data. The profiles of $u_{r m s}$ in figure $10(\mathrm{a})$ and $v_{r m s}$ in figure 10(b) generally indicate over predictions from the calculation, which corresponds to the wider axial velocity profiles in figure 9. Liou et al. ${ }^{41}$ and Inoue ${ }^{42}$ also reported large overpredictions in the turbulence intensities for planar mixing layers. With the confinement of the current threedimensional calculations to a very small domain in the $z$ direction. the inability to calculate large scale fluctuations in this direction may be responsible for the overpredictions of $u_{r m s}$ and $v_{r m s}$. In addition, the Goebel-Dutton Schlieren photographs indicated a very fine turbulent structure contained within the larger scale development, while the LES calculations inher-

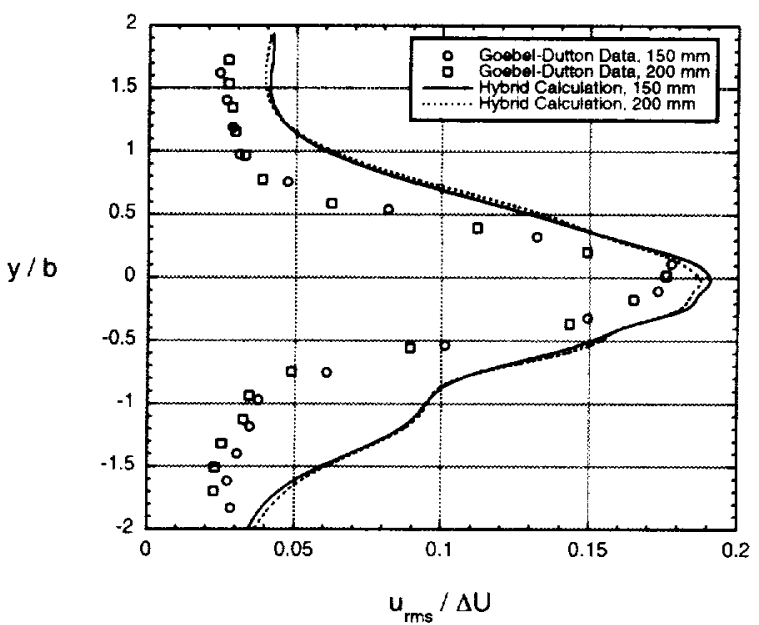

a) $u_{r m s}$

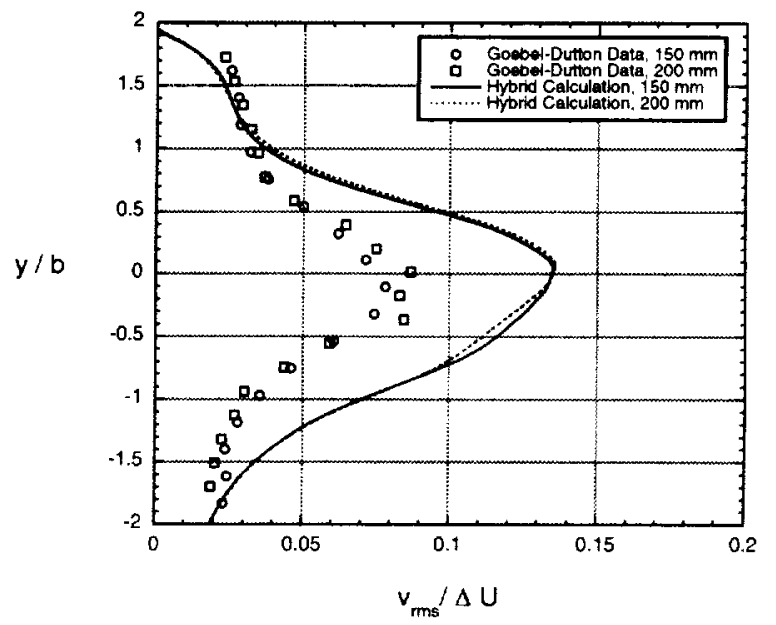

b) $v_{r m}$

Fig. 10 Turbulence intensities in the mixing section

ently can resolve only the larger turbulent scales.

The last three-dimensional case was run using 800 axial points in the mixing section. The finer grid used reduced the permissible time step by nearly a factor of two relative to the 400 axial point case. This reduced time step and doubling the number of grid points in the mixing section would require a factor of four increase in the computer CPU time requirements to run this case to completion, relative to the case with 400 axial points. Considering that the 400 axial point case required $500 \mathrm{CPU}$ hours on a Cray C90 computer, 2000 Cray C90 hours would be required to complete the case with 800 axial grid points. As a result, this last three-dimensional case was run long enough to allow the flow to fully develop in the mixing section, but not long enough to enable time averaging of the turbulent statistics.

In contrast to the grid refinement studies performed for the two-dimensional cases, the large scale turbulent 
development did not change significantly when increasing the number of axial points in the three-dimensional computations from 400 to 800 . The instantaneous density contours in figures 11 indicate a large scale turbulent structure which closely resembles those of the 400 axial grid point case. In particular, the breakdown of the organized vortex structure to turbulence is very similar to those previously shown for the 400 axial grid point case. Within the large scale structures, more fine scale turbulence is evident in the 800 axial point case. This behavior corresponds directly with the philosophy of LES in that as the computational grid is refined, smaller structures are able to be resolved and the role of the subgrid scale model is reduced. In the idealized limit of a grid which is sufficiently fine to resolve all turbulent length scales, a direct numerical simulation is obtained.

\section{Conclusions}

The work described here represents the initial efforts to develop and evaluate a hybrid RANS-LES method for compressible mixing layer simulations. Although the RANS approach does not provide any unsteady turbulent information to the LES region, the mean flow boundary layer characteristics are provided. The hybrid method was developed for the analysis of nozzle and mixing layer configurations in which a dominant structural feature, such as the base region of a nozzle or splitter plate separating the upstream flows, will provide the dominant unsteady mechanism to drive the development of turbulence in the mixing laver.

The Cebeci-Smith turbulence model, despite its relatively simple form, was demonstrated to provide accurate calculations of boundary layer flows in the RANS regions that are free of adverse pressure gradients or separations. Further, the use of the CebeciSmith model in conjunction with the Ota-Goldberg wall function enabled calculations of supersonic wall boundary layers to nearly the same accuracy as that of the standard approach of integrating the Cebeci-Smith model through the viscous sublayer, while enabling a significantly larger vertical grid spacing near the wall. The wall function approach enabled a continuous computational grid to be used from the RANS to the LES regions, and the method thereby avoided the use of discontinuous grid zones that would have required an interpolation scheme at the RANS-LES interface. In addition, the origins of the Cebeci-Smith RANS turbulence model and the Smagorinsky LES subgrid scale model are both in mixing length theory, and this similar form of the models assisted in code implementation. As a result, the use of a more sophisticated turbulence model to close the RANS equations was found to be unnecessary, provided the RANS regions are restricted to attached, zero pressure gradient wall boundary layer regions.

While true LES calculations require computations in three spatial directions, two-dimensional simulations of a benchmark nixing layer experiment were considered first to address effects of axial grid resolution and splitter plate treatment. The parametric study of axial grid resolution indicated more realistic turbulent development with increasing axial grid density. For all of the cases examined, a vortex shedding was found to originate from the base region of a splitter plate separating the upstream wall bounded regions. For the finest grid examined, the unsteady vortex pattern eventually transitioned to a turbulent structure. The location of this transition, however, was much further downstream than observed in the experiments. Calculations obtained for the case in which the finite thickness splitter base was changed to a sharp tip indicated that the vortex shedding was removed.

Three-dimensional calculations were obtained next for grids constructed by copying the two-dimensional planar grids to locations in the third computational direction. Only a small domain was modeled in this third direction, and periodic boundary conditions were employed along the extreme boundaries. For the coarse three-dimensional grid, again no turbulent flow development was observed. For the intermediate grid, the vortex shedding found previously in the two-dimensional simulations was also observed in the three-dimensional calculations. However, the organized vortical structure rapidly disintegrated into a significantly more realistic turbulent flow structure. This rapid transition to turbulent flow was nearly identical to that found in experimental investigations of a similar mixing layer configuration. Although the extent of the third dimension in these calculations was very small, an unsteady mechanism by which disturbances could develop in all three directions and result in a rapid transition to turbulent flow was enabled by the three dimensional calculations. In contrast, a twodimensional approach, by definition, does not allow for such three-dimensional disturbances to develop. The results of these calculations verified that LES simulations must be performed in three dimensions. A final three-dimensional calculation was investigated using a computational grid constructed from the most densely packed two-dimensional grid. Because a prohibitively long run time would be required to complete this solution for turbulent statistics purposes, the calculations were run only long enough to allow the flow to fully develop. The initial transition to turbulence and large scale turbulent structures evident for this case were very similar to those for the intermediate three-dimensional case. More resolution of the finer turbulent scales contained with the larger structures was observed for the fine grid case, which is in line with the philosophy of LES to resolve finer scales as the grid density is increased.

Comparisons of time-averaged axial velocities and turbulence intensities from the calculations to experi- 


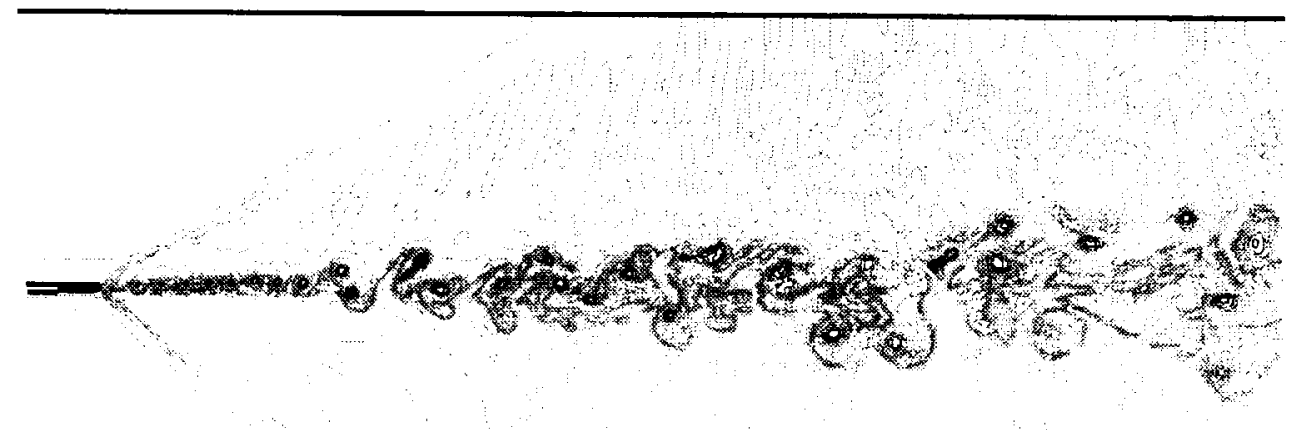

a) Beginning of mixing section

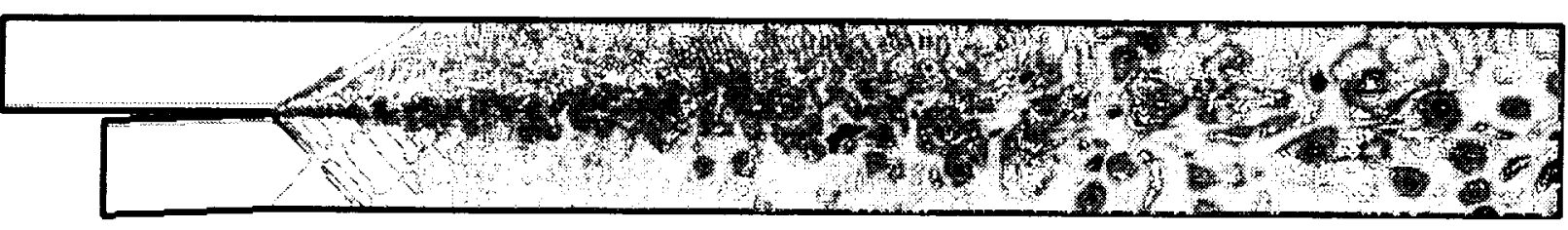

b) Entire mixing section

Fig. 11 Instantaneous density contours for the $3 \mathrm{D}$ case using 800 axial points

mental data indicated reasonable agreement, with the solutions indicating somewhat higher levels of turbulent mixing. A major source of discrepancy between the calculations and experiment is believed to be the lack of adequate grid resolution to resolve the small turbulent scales contained within the larger turbulent structures. Another source of discrepancy was the very small domain used in the third computational direction. Despite these limitations, the three-dimensional calculations demonstrated the success of the hybrid method to capture the dominant characteristics of the mixing layer, and in particular, the rapid transition of the organized vortex structure to a turbulent mixing layer structure. It is expected that improvements in the fidelity of the solution scheme, and more importantly, improvements in computing power, will enable better predictions of the turbulent statistics.

\section{References}

1 J.M. Seiner and E.A. Krejsa. Supersonic Jet Noise and the High Speed Civil Transport. AIAA Paper 89-2358, 1989.

${ }^{2}$ T.J. Barber, L.M. Chiappetta, J.R. DeBonis, N.J. Georgiadis, and D.A. Yoder. An Assessment of Parameters Influencing the Prediction of Shear Layer Mixing. AIAA Journal of Propulsion and Power, 15(1):45-53, 1999.

${ }^{3}$ N.J. Georgiadis, D.A. Yoder, and J.R. DeBonis. A Comparison of Three Navier-Stokes Solvers for Exhaust Nozzle Flowfields. AIAA Paper 99-0748, 1999.

${ }^{4} S . F$. Birch. The Role of Structure in Turbulent Mixing. AIAA Paper 97-2636, 1997.

${ }^{5}$ P. Bradshaw. Turbulence: The Chief Outstanding Difficulty of Our Subject. In The Fifth Symposium on Numerical and Physical Aspects of Aerodynamic Flows. California State University, 1993. Stewartson Memorial Lecture.
${ }^{6}$ S.A. Ragab and S.-C. Sheen. An Investigation of FiniteDifference Methods for Large-Eddy Simulation of a Mixing Layer. AIAA Paper 92-0554, 1992.

'S.A. Ragab and S.-C. Sheen. Large Eddy Simulation of Mixing Layers. In B. Galerpin and S.A. Orszag, editors, Larye Eddy Simulation of Complex Engineering and Geophysical Flous, pages 255-285. Cambridge University Press, 1993.

${ }^{8}$ L.S. Hedges and S. Eberhardt. Numerical Simulation of Total Temperature Separation in Jets. AIAA Paper 92-0535, 1992.

${ }^{9} \mathrm{P}$. Bradshaw. The Effect of Initial Conditions on the Development of a Free Shear Layer. Journal of Fluid Mechanics, 26:225-236. 1966.

${ }^{10}$ F.K. Browand and B.O. Latigo. Growth of the TwoDimensional Mixing Layer from a Turbulent and Nonturbulent Boundary Layer. Physics of Fluids, 22(6):1011-1019, 1979.

${ }^{11}$ A.K.M.F. Hussain and A. R. Clark. Upstream Influence on the Near Field of a Plane Turbulent Jet. Physics of Fluids, 20(9):1416-1425, 1977.

${ }^{12}$ P.R. Spalart, W.H. Jou, M. Strelets, and S.R. Allmaras. Comments on the Feasibility of LES for Wings, and on a Hybrid RANS/LES Approach. In First AFOSR International Conference on DNS/LES, 1997.

${ }^{13}$ P.R. Spalart. Trends in Turbulence Treatments. AIAA Paper 2000-2306, 2000.

${ }^{14}$ P.R. Spalart. Strategies for Turbulence Modeling and Simulations. International Journal of Heat and Fluid Flow, $21(3): 252-263,2000$

${ }^{15}$ P.R. Spalart and S.R. Allmaras. A One-Equation Turbulence Model for Aerodynamic Flows. La Recherche Aerospatiale, 1(1):5-21, 1994.

${ }^{16}$ G.S. Constantinescu and K.D. Squires. LES and DES Investigations of Turbulent Flow over a Sphere. AIAA Paper 2000-0540, 2000.

${ }^{17}$ C.G. Speziale. Turbulence Modeling for Time-Dependent RANS and VLES: A Review. AIAA Journal, 36(2):173-184, 1998.

${ }^{18} \mathrm{P}$. Batten, U. Goldberg, and S. Chaknavarthy. Sub-Grid 
Turbulence Modeling for Unsteady Flow with Acoustic Resonance. AIA A Paper 2000-0473, 2000.

${ }^{19}$ S. Arunajatesan, N. Sinha, and S. Menon. Toward Hybrid LES-RANS Computations of Cavity Flowfields. AIAA Paper 2000-0401, 2000.

${ }^{20}$ G. Erlebacher, M.Y. Hussaini, C.G. Speziale, and T. A. Zang. Toward the large-Eddy Simulation of Compressible Turbulent Flows. NASA CR 187460, 1990. see also ICASE Report 90-76.

${ }^{21}$ C.C. Nelson, Simulations of Spatially Evolving Compressible Turbulence lising a Local Dynamic Subgrid Model. PhD thesis. Georgia Institute of Technology, 1997.

${ }^{22} \mathrm{~T}$. Cebeci. Calculation of Compressible Turbulent Boundary Layers with Heat and Mass Transfer. AIAA Paper 70-741. 1970.

${ }^{23}$ T. Cebeci and A.M.O. Smith. Analysis of Turbulent Boundary Layers. Academic Publishers, 1974.

${ }^{24}$ D.K. Ota and U.C. Goldberg. Law of the Wall with Consistent No-Slip Limit. AIAA Paper 97-2246, 1997.

${ }^{25}$ F.M. White and G.H. Christoph. A Simple Analysis of Compressible Turbulent Two-Dimensional Skin Friction Under Arbitrary Conditions. AFFDL Technical Report 70-133, 1970.

${ }^{26}$ F.M. White. Viscous Fluid Flow. McGraw-Hill, 1974.

${ }^{27} \mathrm{~J}$. Smagorinsky. General Circulation Experiments with the Primitive Equations. Monthly Weather Revieu, 91:99-164. 1963.

${ }^{28}$ P.S. Klebanoff. Characteristics of Turbulence in a Boundary Layer with Zero Pressure Gradient. NACA Technical Note 3178, 1954

${ }^{29}$ P.S. Klebanoff. Characteristics of Turbulence in a Boundary Layer with Zero Pressure Gradient. NACA Report 1247, 1955.

${ }^{30}$ R. Avva, C. Smith, and A. Singhal. Comparative Study of High and Low Reynolds Number Version of k-e Models. AIAA Paper 90-0246, 1990.

${ }^{31}$ R.S. Rogallo and P. Moin. Numerical Simulation of Turbulent Flows. Annual Review of Fluid Mechanics, 17:99-137, 1984.
${ }^{32}$ D. Choi, T.J. Barber, and L.M. Chiappetta. Large Eddy Simulation of High-Reynolds Number Jet Flows. AIAA Paper 99-0230, 1999.

${ }^{33} \mathrm{D}$. Gottlieb and E. Turkel. Dissipative Two-Four Methods for Time-Dependent Problems. Mathematics of Computation. 30(136):703-723, 1976.

${ }^{34}$ D.A. Hudson and L.N. Long. Time Accurate Application of the MacCormack 2-4 Scheme on Massively Parallel Computers. In ICASE/LaRC Workshop on Benchmark Problems in Computational Aeroacoustics, pages 209-215, 1995.

35 A. Bayliss, P. Parikh, L. Maestrello, and E. Turkel. A Fourth-Order Scheme for the Unsteady Compressible NavierStokes Equations. NASA CR 177994, 1985.

${ }^{36}$ S. G. Goebel. An Experimental Investigation of Compress. ible Turbulent Mixing Layers. PhD thesis, University of Illinois at Urbana-Champaign. 1990.

${ }^{37} \mathrm{~S}$. G. Goebel and J.C. Dutton. Velocity Measurements of Compressible Turbulent Mixing Layers. AlAA Paper 90-0709, 1990.

${ }^{38}$ S. G. Goebel and J.C. Dutton. An Experimental Study of Turbulent Compressible Mixing Layers. AIAA Journal. $29(4): 538-546,1991$.

${ }^{39} \mathrm{M}$. Tucker. Approximate Calculation of Turbulent Boundary-Layer Development in Compressible Flow. NASA Technical Note 2337, 1951.

${ }^{40}$ N.T. Clemens and M.G. Mungal. Two and ThreeDimensional Effects in the Supersonic Mixing Layer. AIAA Journal, 30(4):973-981, 1992.

41 T.M. Liou. W.Y. Lien, and P.W. Hwang. Compressibility Effects and Mixing Enhancement in Turbulent Free Shear Flows. AIAA Journal, 33(12):2332-2338, 1995.

$42 O$. Inoue. Vortex Simulation of a Turbulent Mixing Layer AIAA Journal, 23(3):367-373, 1985. 


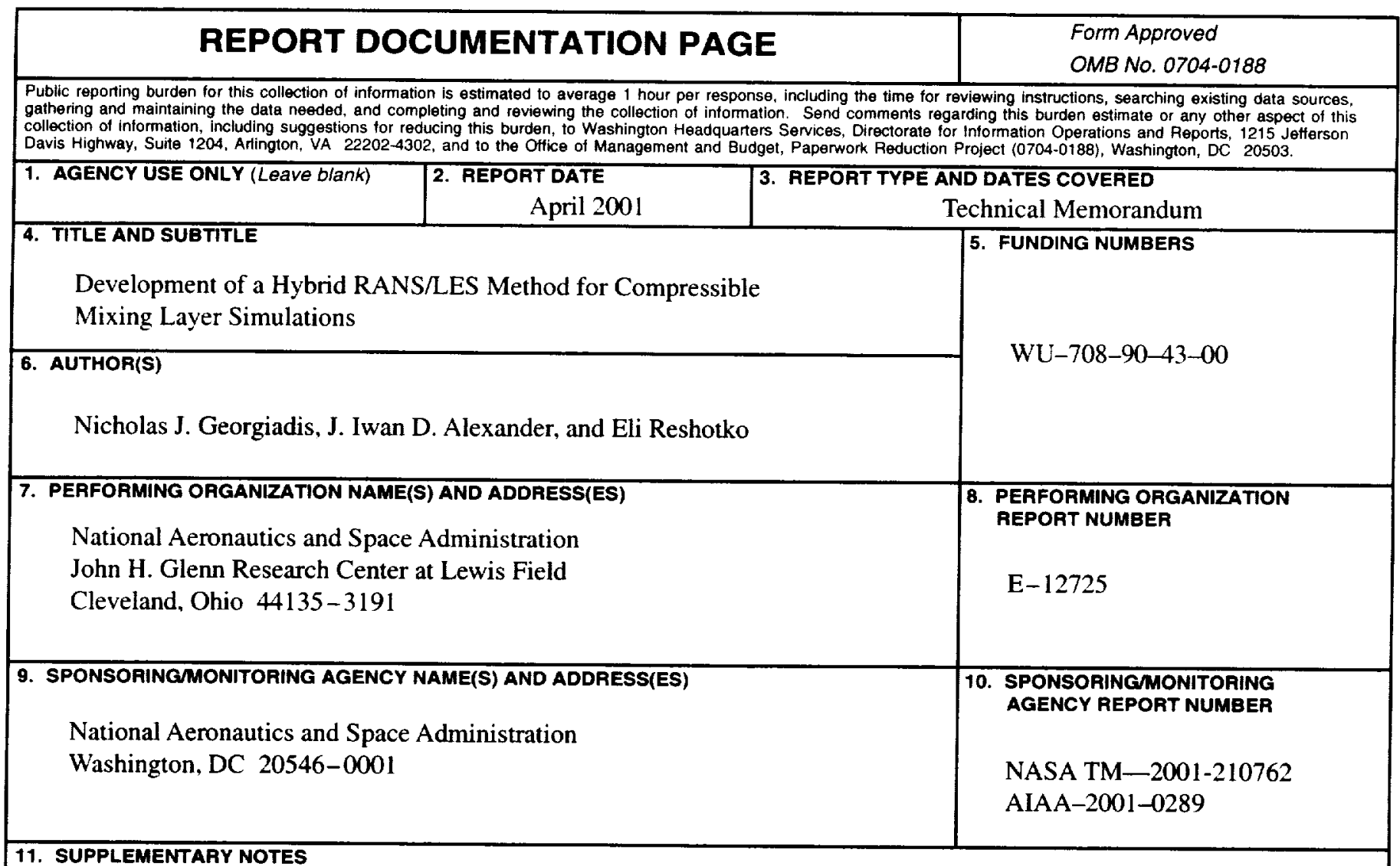

Prepared for the 39th Aerospace Sciences Meeting and Exhibit sponsored by the American Institute of Aeronautics and Astronautics, Reno, Nevada, January 8-11, 2001. Nicholas J. Georgiadis, NASA Glenn Research Center; J. Iwan D. Alexander and Eli Reshotko, Case Western Reserve University, Cleveland, Ohio 44106. Responsible person, Nicholas J. Georgiadis, organization code 5860, 216-433-3958.

12a. DISTRIBUTION/AVAILABILITY STATEMENT

Unclassified - Unlimited

Subject Categories: 02 and 34

Distribution: Nonstandard

Available electronically at http://gitrs.grc.nasa.gov/GLTRS

This publication is available from the NASA Center for AeroSpace Information, 301-621-0390.

13. ABSTRACT (Maximum 200 words)

A hybrid method has been developed for simulations of compressible turbulent mixing layers. Such mixing layers dominate the flows in exhaust systems of modern day aircraft and also those of hypersonic vehicles currently under development. The hybrid method uses a Reynolds-averaged Navier-Stokes (RANS) procedure to calculate wall bounded regions entering a mixing section, and a Large Eddy Simulation (LES) procedure to calculate the mixing dominated regions. A numerical technique was developed to enable the use of the hybrid RANS-LES method on stretched, nonCartesian grids. The hybrid RANS-LES method is applied to a benchmark compressible mixing layer experiment. Preliminary two-dimensional calculations are used to investigate the effects of axial grid density and boundary conditions. Actual LES calculations, performed in three spatial directions, indicated an initial vortex shedding followed by rapid transition to turbulence, which is in agreement with experimental observations.

\section{SUBJECT TERMS}

Turbulent mixing; Nozzle; Compressibility; Large eddy simulation; Navier-Stokes

\begin{tabular}{|c|c|c|}
\hline $\begin{array}{c}\text { 17. SECURITY CLASSIFICATION } \\
\text { OF REPORT } \\
\text { Unclassified }\end{array}$ & $\begin{array}{c}\text { 18. SECURITY CLASSIFICATION } \\
\text { OF THIS PAGE } \\
\text { Unclassified }\end{array}$ & $\begin{array}{c}\text { 19. SECURITY CLASSIFICATION } \\
\text { OF ABSTRACT } \\
\text { Unclassified }\end{array}$ \\
\hline
\end{tabular}


. 